\title{
Image Compression using Blind Watermarking Technique
}

\author{
K. Sheikdavood, S. Monish, S. Rajvignesh, P. Poornaprasath, A. Pugalenthi and D. Pandisiva \\ Department of Electronics and Communication Engineering, M. Kumarasamy College of Engineering, \\ Thalavapalyam, Karur -639113, Tamil Nadu, India; sheikdavoodk.ece@mkce.ac.in, monishsengodan@gmail.com, \\ rajvignesh11111@gmail.com, pooranaprasath1@gmail.com, pugalenthiece@gmail.com, pandisivabe@gmail.com
}

\begin{abstract}
Objectives: A new watermarking idea is projected that would impressively enhance present day watermarking practices. This idea endeavors the highlights of minor images of watermarks of the standard image. This will guide us to maintain secrete content and images in the social media. This will safeguard us from unwanted hackers. Method of Analysis: To make connection methodology and Similar irrelevant images through fuzzy rules are grouped or might be produced using the host image to simulate an extracted watermark. This technique, as the feature classification, forest, can do dazzle withdrawal and variable to any watermarking topic utilizing a quantization-based module. In addition, a greater extent, a watermark is acknowledged while an incompatible influence on the physical property of the cover image. Findings: The tests show the profitable re-enactment of watermarks and furthermore the application to surprising watermarking plans. One among them features classification, forest marginally balanced from a connection to especially opposing JPEG pressure, and furthermore, the authors demonstrate local benefits of the SVD adjustment method to oppose very surprising image process. Application/Improvements: To maintain personal information secured.
\end{abstract}

Keywords: Features Classification Forest, Gray Level Co-Occurrence Matrix, Single Value Decomposition

\section{Introduction}

The anticipated idea fuses a model, to be specific, the Features Classification Forest, that extensively enhances the ability of visually impaired watermarking frameworks without the symptoms of corrupting the physical property and quality, and it will be redone to those watermarking methods upheld numerical property change or on the other hand a division system. These two courses here imply that a twofold arrangement will be installed by controlling a gathering of the properties of a picture in a methodical way to get a perceived condition inside which each property speaks to exclusively whichever an absolute or opposing approach, that the parallel grouping the absolute approach remains for bit ${ }^{1}$, and furthermore the opposing approach remains for bit 0 will be implanted into the cover image.

\subsection{Features Classification Forest}

The main topic utilizes the CRT hypothesis in light of the fact that the adjustment controls and put on the different trigonometric cosine change on an $8 \times 8$ estimated square. $\mathrm{A} D \mathrm{DC}$ and three $\mathrm{AC}$ quantities are picked on the grounds that the inserting zone to install the watermark bit flow ${ }^{1}$. The topic is included via the protection of JPEG pressure; at the same time, it's to some degree weaker than the fifth idea, which can be appeared inside the investigation area. The second idea applies (SVD) on a $4 \times 4$ measured square 2 . By moving investigation of the connections of parts inside the $U$ orthogonal lattice, the topic found that the components set at the subsequent line introductory segment and in this manner the third line starting section are next to each option ${ }^{2}$. Consequently, the watermark bit flow is inserted into the connection of those dual sections by modifying any one in everything about components to be bigger than the inverse in Figure 1.

${ }^{*}$ Author for correspondence 
Those plans take the change or quantization component in light of the fact that the performance to present the water mark bit flow, by either altering the connection among numerous possessions inside the cover image or delicately modifying the components inside the network to accomplish the Figure 1, implanting reason. The greater part of the visually impaired advanced watermarking strategies receives a comparative style rationality ${ }^{3}$. The FCT is overwhelmed by affiliation analysis that was initially connected to the forecast of the customer seeking tendencies. At the point, when certain smaller scale images have the same connection rules, it concludes that the appearance and surface of these smaller scale images are fundamentally the same as Figure 2. Few images from each the cover image and watermark as item and adventures the affiliation controls by this image ${ }^{4}$. At the point when bound little images have a comparable affiliation rule, it infers that the looks and surface of those little images are frightfully comparable. From this thought, we tend to utilize the affiliation runs as graphic limbs to build the tree assemblies. To make a worthy model, the highlights of the images ought to be framed to appropriately set up the affiliation directions.
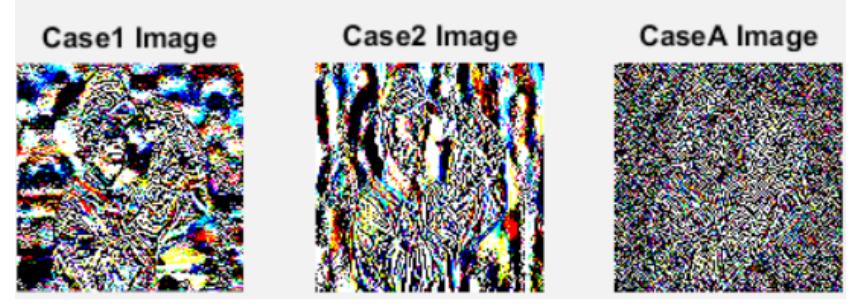

Figure 1. Features Classification Forest of Cover Image.
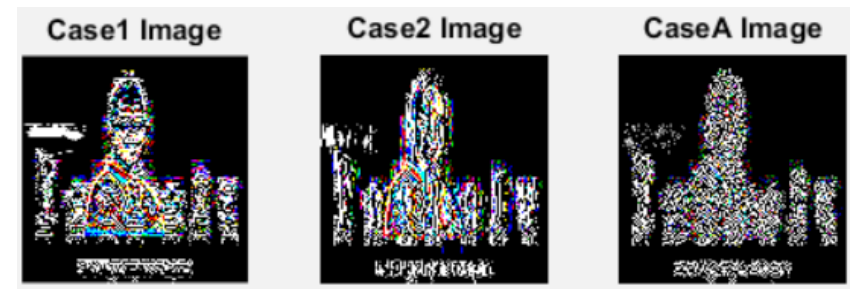

Figure 2. Features Classification of Secret Image

\subsection{Image Analysis}

An image is included a curiously huge scope of pixels. Everyone of the pixels itselfexclusively demonstrates its force. Joining a specific scope of pixels along can bring about a scope of various combos that may create particular surfaces, similar to creature hides or animal skin. Subsequently, on the off chance that we tend to change the primary attention from a complete digital image into a little extent of minor pixel blends, the likenesses can perform on very surprising images ${ }^{5}$.

\subsection{Gray-Level Spatial Dependence Matrix}

A measurable strategy for looking considerations of the spatial relationship of pixels is the gray-level spatial dependence matrix, otherwise called the gray-level cooccurrence matrix (GLCM) ${ }^{6}$. The GLCM volumes define the superficial of an image by supposing how recurrently associations of the pixel with particular approvals and in a predefined spatial connection happens while making a GLCM and afterward eliminating the definite methods from this lattice (Figure 3).

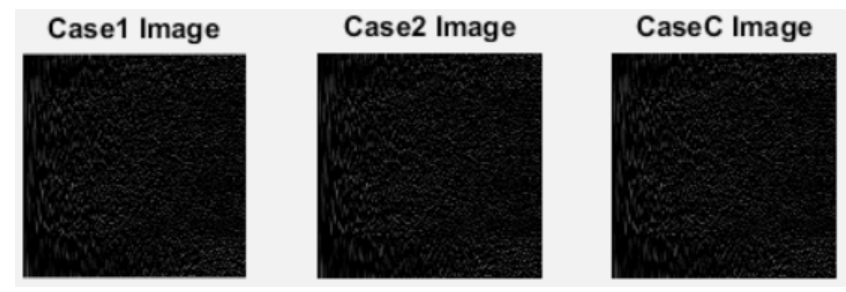

Figure 3. Grey Level Co-Occurrence of Cover Image.

\section{Watermark Embedding and Extraction}

So far, 2 styles of FCTs have been resolved. Thereafter, a new proposed inserting topic upheld is presented completely. Afterward, the flowchart of the whole watermarking topic can take over.

\subsection{SVD Adjustment Method}

In SVD advanced watermarking procedures, the most attribute are that solitary esteems protect the shine level of the image square, and particular vectors safeguard the geometric properties of the image piece. An inside and out an examination of the aftereffects of different assaults is given ${ }^{\underline{z}}$. On the off chance that the adjustment of the solitary esteem isn't durable, at that point the brilliance of the picture square won't change clearly; therefore, we can manufacture utilization of this trademark to present a paired piece while not touching the physical property in Figure 4. 


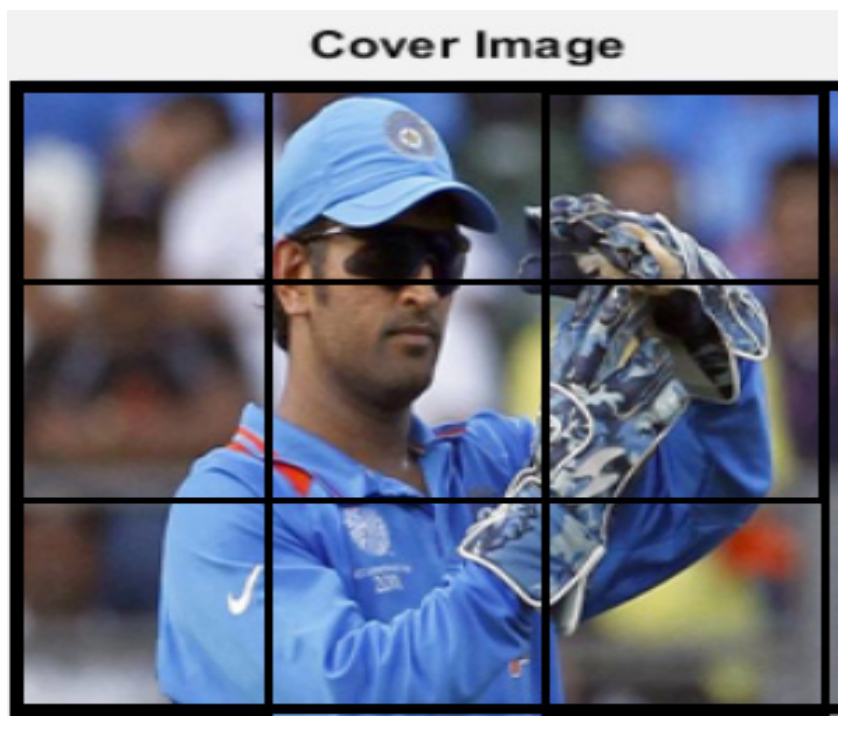

Figure 4. Cover Image.

Toward the beginning of the inserting stream, the coveted watermark is partitioned into $\mathrm{k} \times \mathrm{k}$ measured pieces and each square experiences the FCT to search out the good affiliation run the show. Then again, the host image is partitioned into $\mathrm{K} \times \mathrm{K}$ estimated squares at that point in Figure 5, the greater part of the implanting area ${ }^{8}$. Next, the paired flow of the watermark and in this way the solitary esteems from the host image meet in the gray space spoke to inside the figure. The secret's a self-styled irregular range generator to mastermind the request of the particular esteems, and each and every piece of the double stream is inserted into one solitary incentive in a systematic way by the changing instrument said inside the past subdivision. For now, the installing technique finishes and along these lines the watermarked image is so made. For the extraction stream, the implanted affiliation rules are coming back from the watermarked image exclusively by the opposite rationale of the installing part. The watermarked image is initially separated afresh into $\mathrm{K} \times \mathrm{K}$ estimated squares, and particular esteem decay is utilized a short time later and connected to or the majority of the pieces. A parallel stream is legitimately recovered by basic leadership regardless of whether the deposit of the modulo activity on the particular cost is any bigger than the average value. Along these lines, the affiliation rules are recovered from the recovered twofold stream in Figure 5.

Up to this point, the focuses communicated over are clear because of them are like the inserting stream how-

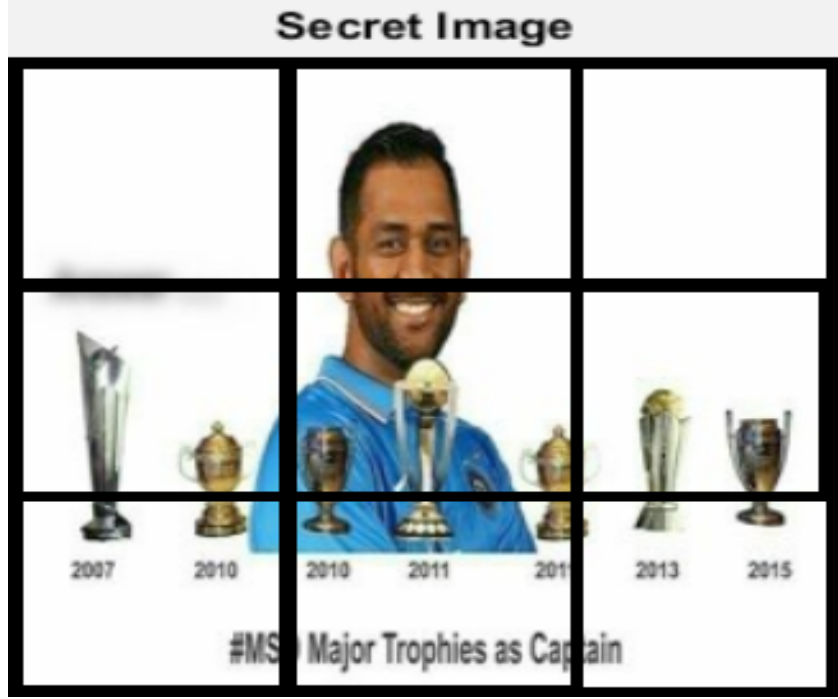

Figure 5. Secret Image.

ever backward. Notwithstanding, the statements a short time later are extraordinary, and accordingly the center cost of the FC Forest is repeated totally 9 . The watermarked image, at the consistent period, is isolated into $\mathrm{k} \times \mathrm{k}$ measured squares. They're not characterized by the inquiry of affiliation rules $\frac{10}{}$, however, keep inside the comparing classifications determined the returned affiliation standards will utilize them as substitutions to re-enact the pieces of the underlying watermark $\underline{11}$. At that point, each affiliation manages to utilize the plan to require up and around the squares of the watermarked image into the relating session and ascertain their normal as a substitution $\mathrm{k} \times \mathrm{k}$ measured piece, to set up the watermarked ${ }^{12}$. As of recently, the withdrawal stream separates a substitution watermark that is reunited by the affiliation directions.

\section{Experimental Results}

To review the exploratory outcomes, the estimations to review two principles, the physical property, and quality, square measure presented in Figure 6. Once the cover image executes the watermarking technique, we acquire. The watermarked picture. At the point when the extraction section, a spic and span watermark recreates the main watermark by the FCT. At the point when the withdrawal, the watermark that is originated from the cover image has no doubt altered. In this manner, a measure is critical. All in all, once the NCC is greater than 0.9, the separated watermark will at present be 
perceived Figure 6. As we tended to specify already, the most objective of the arranged idea is to broaden the capacity while not including the physical property and quality. In any case, there's an exchange off among the 3 criteria, once forcing one paradigm, that model would affect the others. Consequently, we tend to make utilization of specially focused on assessing the best approach to adjust the exchange offs on computerized watermarking plans.

\section{Conclusion}

Inside the starting, the trade among the 3 criteria, the impalpability, strength, and volume, is given to elucidating the most points of digital watermarking systems. To make headway on the far side these constraints, the idea of misuse few images privileged the cover image to reproduce a watermark is overwhelmed from affiliation directions and subsequently encourages the production of the Features Classification Forest. The exploratory outcomes at first demonstrate the ability change in the different plans. The second trial displays the importance and inborn quality underneath numerous assaults. Next, we tend to demonstrate that the two sorts of FCTs manage different watermarks fine. Inside the last trial, the connection dispatch between the physical property and quality keeps up a trade that will be that the premier perplexity and test that scientists wish to be at.

Overall, the FCT gives a substitution advancement to enhance the capability while not cooperating the physical property and strength. This methodology will create digital watermarking techniques a lot of versatility to users owing to visual impairment, larger capability, and no sacrifice of physical property and strength, and it's a lot of sense due to its relevance to most of the opposite techniques. By using this idea, a lot of totally different techniques may be made-up or custom-made to enhance the capability, thereby providing a lot of accessibility for users as a result of the dimensions of the watermark had better be a lot of flexibility in its sensible utilize.

\section{References}

1. Andalibi M, Chandler DM. Digital image watermarking via adaptive logo texturization. IEEE Transactions on Image Processing. 2015 December; 24(12):5060-73. Crossref. PMid:26353371

2. Sheikdavood K, Surendar P. A Review: Undetectable Watermarking in Printed Images. International Journal of Pure and Applied Mathematics. 2018 February; 118(18):3077-83.

3. Horng SJ, Rosiyadi D, Li T, Takao T, Guo M, Khan MK. A blind image copyright protection scheme for e-government. Journal of Visual Communication and Image Representation. 2013; 24(7):1099-105. Crossref.

4. Palanivel Rajan S, Sheikdavood K. Performance Evaluation on Automatic Follicles Detection in the Ovary. International Journal of Applied Engineering Research. 2015; 10(55):1-5.

5. Sheikdavood K, Arulpriya S. Analysis of Saliency Recognition through Comparable Image Recovery. Journal of Chemical and Pharmaceutical Sciences. 2016; 8:108-112.

6. Patra C, Phua J E, Bornand C. A novel DCT domain CRTbased watermarking scheme for image authentication surviving JPEG compression. Digital Signal Processing. 2010; 20(6):1597-611. Crossref.

7. Sheikdavood K, Palanivel RS. Analysis of Ovarian Diseases Using Ultrasound Images. Journal of advances in chemistry. 2016; 12(10):4449-54.

8. Su Q, Niu Y, Wang G, Jia S, Yue J. Color image blind watermarking scheme based on QR decomposition. Signal Process. 2014; 94:219-35. Crossref.

9. Sheikdavood K, Mahamudha P, Nagendran K. Performance Evaluation on Accurate Coronary Centerline Extraction and Catheter Detection in Angiographies. International Journal of Applied Engineering Research. 2015; 10(1):349-53.

10. Su Q, Niu Y, Zou H, Liu X. Blind dual color images watermarking based on singular value decomposition. Applied Mathematics and Computation. 2013; 219(16):8455-66. Crossref.

11. Sheikdavood K, Surendar P, Manikandan A. Certain Investigation on Latent Fingerprint Improvement through Multi-Scale Patch Based Sparse Representation. Indian Journal of Engineering. 2016; 13(31):59-64.

12. Zhu X, Ding J, Dong H, Hu K, Zhang X. Normalized correlation-based quantization modulation for robust watermarking, IEEE Transactions on Multimedia. 2014; 16(7):1888-1904. Crossref. 